PHYSICAL REVIEW E 71, 056129 (2005)

\title{
Exact dynamics of a reaction-diffusion model with spatially alternating rates
}

\author{
M. Mobilia, ${ }^{*}$ B. Schmittmann, ${ }^{\dagger}$ and R. K. P. Zia \\ Center for Stochastic Processes in Science and Engineering, Department of Physics, Virginia Tech, \\ Blacksburg, Virginia 24061-0435, USA
}

(Received 21 December 2004; published 31 May 2005)

\begin{abstract}
We present the exact solution for the full dynamics of a nonequilibrium spin chain and its dual reactiondiffusion model, for arbitrary initial conditions. The spin chain is driven out of equilibrium by coupling alternating spins to two thermal baths at different temperatures. In the reaction-diffusion model, this translates into spatially alternating rates for particle creation and annihilation, and even negative "temperatures" have a perfectly natural interpretation. Observables of interest include the magnetization, the particle density, and all correlation functions for both models. Two generic types of time dependence are found: if both temperatures are positive, the magnetization, density, and correlation functions decay exponentially to their steady-state values. In contrast, if one of the temperatures is negative, damped oscillations are observed in all quantities. They can be traced to a subtle competition of pair creation and annihilation on the two sublattices. We comment on the limitations of mean-field theory and propose an experimental realization of our model in certain conjugated polymers and linear chain compounds.
\end{abstract}

DOI: 10.1103/PhysRevE.71.056129

PACS number(s): 02.50.-r, 75.10.-b, 05.50.+q, 05.70.Ln

\section{INTRODUCTION}

Nonequilibrium many-body systems abound in the physical and life sciences and have recently received much attention (see, e.g., [1-3] and references therein). Despite these efforts, a comprehensive theoretical framework is still lacking: As yet, there is no equivalent of Gibbs ensemble theory for nonequilibrium systems. As a consequence, in contrast to equilibrium statistical mechanics, macroscopic observables cannot be computed without explicit reference to the imposed dynamics, generally described by a master equation, and most progress in the field is made by studying paradigmatic models [2]. In this context, exact solutions of simple models are scarce, but very precious, since they can serve as testing grounds for approximate and/or numerical schemes and shed light on general properties of whole classes of related models. Not surprisingly, nontrivial solutions are almost entirely restricted to one dimension (1D; see, e.g., $[2,3])$, and have focused on completely uniform lattices with site-independent rates. Clearly, however, one would like to take into account more complex situations, e.g., those with spatially varying coupling constants or rates. Arguably, one of the simplest generalizations beyond a completely uniform system is one with alternating rates. In the following, we consider a 1D kinetic Ising chain (KISC), coupled to two alternating temperatures and endowed with Glauber-like dynamics. Our analysis of this model provides a full description of its dual counterpart, namely a reaction-diffusion system (RDS), characterized by spatially alternating annihilation and creation rates. Members of these two classes-i.e., kinetic Ising and reaction-diffusion modelsare prototypical nonequilibrium systems which have been

\footnotetext{
*Electronic address: mmobilia@vt.edu

${ }^{\dagger}$ Electronic address: schmittm@vt.edu

Electronic address: rkpzia@vt.edu
}

thoroughly studied on homogeneous lattices [2-7]. Yet they still offer surprises and novel behaviors, when nontrivial spatial rates are investigated.

Our model was first introduced by Ràcz and Zia [8], who recognized that (stationary) two-point correlation functions are easily found exactly, even though spins on alternating sites are coupled to different temperatures. Schmittmann and Schmüser subsequently realized that all stationary correlation functions are exactly calculable [9]. While this information is equivalent to the full stationary solution, its representation as $\exp \left(-\mathcal{H}_{\mathrm{eff}}\right)$ is nontrivial, involving a proliferation of longer-ranged multispin couplings [10]. Finally, we recently reported the exact solution for all dynamic correlation functions, starting from a very simple initial condition, i.e., zero magnetization and vanishing correlations [11].

In this article, we complete these earlier studies by demonstrating how competing site-dependent rates may dramatically affect the dynamics by giving rise to an oscillatory approach toward the nonequilibrium steady state. We use a generating functional approach to obtain the complete solution for all correlation functions with arbitrary initial conditions. We focus specifically on the dynamical magnetization and the spin-spin correlations and explore their long-time behavior. We will also consider the dynamics of domain walls in the spin chain which can be mapped onto a reactiondiffusion system. Interpreting our results in the language of particle annihilation and creation, negative "temperatures" acquire a natural physical meaning, leading to unexpected oscillatory dynamics. From a more technical point of view, we are able to obtain a complete solution for two nontrivial nonequilibrium many-body systems which provides some insight into the solvability of two whole classes of related models.

The mapping to a reaction-diffusion system is of interest for two reasons. On the theoretical side, the equations for densities and correlation functions in the RDS form an infinite hierarchy whose solution is not at all apparent until one 
TABLE I. Basic processes underlying the KISC (left) and RDS (middle) dynamics.

\begin{tabular}{ccc}
\hline \hline Spin flip of site $j$ & Reactions at bonds next to site $j$ & Rates \\
\hline$+--\rightarrow++-$ and $--+\rightarrow-++$ & $A \varnothing \rightarrow \varnothing A$ and $\varnothing A \rightarrow A \varnothing$ & $1 / 2$ \\
$+-+\rightarrow+++(j$ even $)$ & $A A \rightarrow \varnothing \varnothing(j$ even $)$ & $\left(1+\gamma_{e}\right) / 2$ \\
$+-+\rightarrow+++(j$ odd $)$ & $A A \rightarrow \varnothing \varnothing(j$ odd $)$ & $\left(1+\gamma_{o}\right) / 2$ \\
$+++\rightarrow+-+(j$ even $)$ & $\varnothing \varnothing \rightarrow A A(j$ even $)$ & $\left(1-\gamma_{e}\right) / 2$ \\
$+++\rightarrow+-+(j$ odd $)$ & $\varnothing \varnothing \rightarrow A A(j$ odd $)$ & $\left(1-\gamma_{o}\right.$ \\
\hline \hline
\end{tabular}

recognizes the equivalent spin chain model. Also, from an experimental perspective, it is well known that diffusionlimited reactions with annihilation and creation of pairs of particles are good models for the photogrowth properties of excited states (solitons/antisoliton pairs) in certain conjugated polymers and linear chain compounds [12-14]. We propose that spatially alternating creation/annihilation rates in these systems-especially in MX chain compounds-can be generated with the help of a laser with spatially modulated power output.

This article is organized as follows. In the next section, we introduce the kinetic spin chain and its dual reactiondiffusion system. Section III presents the complete solution of the spin chain. Some technical details are relegated to two Appendixes. In Sec. IV, we map the two-temperature spin chain onto a reaction-diffusion system with alternating rates, whose density and correlation functions are computed. We analyze the conditions under which damped oscillations characterize the approach to the steady state, and we compare our exact results to a simple mean-field description. Section $\mathrm{V}$ is devoted to a brief discussion of the solvability of related models, with Sec. VI reserved for our conclusions.

\section{THE MODELS}

We consider two closely related nonequilibrium manyparticle systems on a one-dimensional lattice: (i) a kinetic Ising spin chain (KISC) endowed with a generalized Glauber-like dynamics, and (ii) a reaction-diffusion system (RDS), with spatially periodic pair annihilation and creation rates. For convenience, we restrict ourselves to a periodic lattice (a ring) with an even number of sites and study the thermodynamic limit. We expect our exact results to be valid for the general cases of an odd number of sites and/or arbitrary boundary conditions, apart from the usual caveats.

Since the RDS follows from the spin chain via a duality relationship, we focus mainly on the detailed description of model (i). A spin variable, $\sigma_{j}= \pm 1$, denotes the value of the spin at site $j$, with $j=1,2, \ldots, L$, and $L$ an even integer. Nearest-neighbor spins interact according to the usual Ising Hamiltonian: $\mathcal{H}=-J \Sigma_{j} \sigma_{j} \sigma_{j+1}$, where $J>0(J<0)$ is the (anti)ferromagnetic exchange coupling. Our model is endowed with a nonequilibrium generalization of the usual Glauber [5] dynamics: spins on even and odd sites experience different temperatures, $T_{e}$ and $T_{o}$, which implies the violation of detailed balance [8-10]. To be specific, a configuration $\left\{\sigma_{1}, \sigma_{2}, \ldots, \sigma_{L}\right\}$ evolves into a new one by random sequential spin flips: A spin $\sigma_{j}$ flips to $-\sigma_{j}$ with rate

$$
w_{j}(\{\sigma\}) \equiv w_{j}\left(\sigma_{j} \rightarrow-\sigma_{j}\right)=\frac{1}{2}-\frac{\gamma_{j}}{4} \sigma_{j}\left(\sigma_{j-1}+\sigma_{j+1}\right),
$$

where $\gamma_{2 i}=\gamma_{e}=\tanh \left(2 J / k_{b} T_{e}\right)$ and $\gamma_{2 i+1}=\gamma_{o}=\tanh \left(2 J / k_{b} T_{o}\right)$, on even $(j=2 i)$ and odd $(j=2 i+1)$ sites. The time-dependent probability distribution $P(\{\sigma\}, t)$ obeys the master equation

$$
\partial_{t} P(\{\sigma\}, t)=\sum_{j}\left[w_{j}\left(\{\sigma\}^{j}\right) P\left(\{\sigma\}^{j}, t\right)-w_{j}(\{\sigma\}) P(\{\sigma\}, t)\right],
$$

where the state $\{\sigma\}^{j}$ differs from $\{\sigma\}$ only by the spin flip of $\sigma_{j}$. Our main goal in this work is to compute the timedependent distribution $P(\{\sigma\}, t)$. To do so, we compute all correlation functions $\left\langle\sigma_{j_{1}} \cdots \sigma_{j_{n}}\right\rangle_{t} \equiv \Sigma_{\{\sigma\}} \sigma_{j_{1}} \cdots \sigma_{j_{n}} P(\{\sigma\}, t)$ and invoke the following relationship [5]:

$$
\begin{aligned}
2^{L} P(\{\sigma\}, t)= & 1+\sum_{i} \sigma_{i}\left\langle\sigma_{i}\right\rangle_{t}+\sum_{j>k} \sigma_{j} \sigma_{k}\left\langle\sigma_{j} \sigma_{k}\right\rangle_{t} \\
& +\sum_{j>k>l} \sigma_{j} \sigma_{k} \sigma_{l}\left\langle\sigma_{j} \sigma_{k} \sigma_{l}\right\rangle_{t}+\cdots
\end{aligned}
$$

This expression illustrates that the knowledge of all equaltime correlation functions is equivalent to the complete knowledge of the distribution function $P(\{\sigma\}, t)$. Recently, this implication was exploited for the steady state [9], and for the time-dependent situation yet restricted to a particularly simple initial condition [11].

The spin-flip dynamics of this Ising chain can be expressed in terms of the creation, annihilation, and diffusion of domain walls, i.e., pairs of spins with opposite sign. For example, flipping $\sigma_{j}$ in the local configuration $\sigma_{j-1}=\sigma_{j}$ $=\sigma_{j+1}=+1$ creates two domain walls: $\sigma_{j-1}=-\sigma_{j}$ and $\sigma_{j}=$ $-\sigma_{j+1}$, located on the bonds $(j-1, j)$ and $(j, j+1)$. Similarly, flipping $\sigma_{j}$ in the local configuration $\sigma_{j-1}=\sigma_{j}=-\sigma_{j+1}=+1$ has the effect of moving the domain wall on bond $(j, j+1)$ by one lattice constant to the left, corresponding to domain-wall diffusion. By identifying a domain wall with a "particle," $A$, our spin-flip dynamics can be recast as a reaction-diffusion model, and the two examples translate into $\varnothing \varnothing \rightarrow A A$ and $\varnothing A \rightarrow A \varnothing$, respectively. The mapping from the KISC into its dual RDS is described in detail in Table I.

Clearly, the presence of alternating temperatures $T_{e}, T_{o}$ in spin language translates into alternating pair annihilation and creation rates $\left(1 \pm \gamma_{e, o}\right) / 2$ in the RDS. We can see easily that letting $T_{e}$ or $T_{o}$ vanish simply prohibits pair creation entirely at even or odd sites. Remarkably, we can derive an additional, and possibly rather unexpected, benefit from this 
mapping: Assigning negative values for the temperatures $T_{e}$ and/or $T_{o}$ may appear artificial in the KISC, but is perfectly natural in the RDS: For example, $T_{e}<0$ simply corresponds to a creation rate $\left(1-\gamma_{e}\right) / 2>1 / 2$ which is easily implemented in a simulation. In other words, the RDS version is physically meaningful, and readily accessible, on a much wider parameter space.

\section{COMPLETE SOLUTION OF THE KINETIC SPIN CHAIN}

In this section, we completely solve the dynamics of the KISC. It was shown previously [15] that the generating function, and hence the full distribution $P(\{\sigma\}, t)$, of a broad class of Ising models can be computed from two very basic observables, namely (i) the magnetization $m_{j}(t)=\left\langle\sigma_{j}\right\rangle_{t}$ for $\operatorname{arbi}$ trary initial condition, and (ii) a particular two-point equaltime correlation function, $c_{j, k}(t)=\left\langle\sigma_{j} \sigma_{k}\right\rangle_{t}$, the resultant from the special initial conditions $m_{j}(0)=c_{j, k}(0)=0$ (see Appendix A for a more detailed discussion of this statement). Here, $\langle\bigcirc\rangle_{t} \equiv \sum_{\{\sigma\}} \bigcirc P(\{\sigma\}, t)$ denotes the usual configurational average. In the following, we assemble the necessary information about these two observables.

\section{A. The general $t$-dependent magnetization}

From our earlier work [11], we recall that the magnetization $m_{j}(t)=\left\langle\sigma_{j}\right\rangle_{t}$ of the KISC obeys the equation of motion, $(d / d t) m_{j}(t)=\left(\gamma_{j} / 2\right)\left[m_{j-1}(t)+m_{j+1}(t)\right]-m_{j}(t)$, which is easily derived from the master equation, Eq. (2). As shown in [11], the general solution of this linear equation takes the form $m_{j}(t)=\Sigma_{k} M_{j, k}(t) m_{k}(0)$, where the "propagator" $M_{j, k}(t)$ can be written in term of modified Bessel functions of the first kind $I_{n}(t)[16]$,

$$
M_{j, k}(t)=e^{-t} \sqrt{\frac{\gamma_{j}}{\gamma_{k}}} I_{k-j}(\alpha t) \text { with } \alpha \equiv\left(\gamma_{e} \gamma_{o}\right)^{1 / 2} .
$$

If $\gamma_{e} \gamma_{o}<0$, the propagator becomes $M_{j, k}(t)$ $=i(-1)^{(k-j) / 2}\left|\gamma_{j} / \gamma_{k}\right|^{1 / 2} e^{-t} J_{k-j}(|\alpha| t)[11]$, where $J_{n}(t)$ is a Bessel function of the first kind, with damped oscillatory asymptotic behavior [16]. This translates into an oscillatory decay of the magnetization [11].

\section{B. A special two-point equal-time correlation function}

The second fundamental quantity, i.e., the equal-time spin-spin correlation function $c_{k, j}(t)$, with $k>j$, is already known from [11]. For our purposes, it suffices to consider an initial condition with zero magnetization and zero initial correlations. With the boundary condition $\left\langle\sigma_{j} \sigma_{k}\right\rangle_{t}=1$ for $j=k$, this basic correlation depends only on the distance between the two sites and their parity, $\mu(k), \mu(j) \in\{e, o\}$ [11],

$$
c_{k, j}(t) \equiv c_{k-j}^{\mu(k), \mu(j)}(t)=\frac{\bar{\gamma}}{\alpha^{2}} \sqrt{\gamma_{j} \gamma_{k}}(k-j) \int_{0}^{2 t} \frac{d \tau}{\tau} e^{-\tau} I_{k-j}(\alpha \tau),
$$

where

$$
\bar{\gamma} \equiv\left(\gamma_{e}+\gamma_{o}\right) / 2 .
$$

For long times, these settle into their stationary values $[8,9]$, independent of initial conditions,

$$
\left\langle\sigma_{j} \sigma_{k}\right\rangle_{\infty} \equiv c_{k, j}(\infty)=\frac{\bar{\gamma}}{\sqrt{\gamma_{j-1} \gamma_{k-1}}} \omega^{k-j},
$$

where

$$
\omega \equiv \frac{\alpha}{1+\sqrt{1-\alpha^{2}}},
$$

a quantity that reduces to the familiar $\tanh \left(J / k_{b} T\right)$ in the equilibrium Ising chain. The approach to these values is exponential and monotonic, as $e^{-2(1-\alpha) t} t^{-3 / 2}$, provided $\gamma_{e} \gamma_{o}>0$. However, for $\gamma_{e} \gamma_{o}<0$, the approach is oscillatory and damped by $e^{-2 t} t^{-3 / 2}$ [11]. For later reference, it is convenient to display the parity dependence explicitly. Since translation invariance ensures $c_{k-j}^{o e}(t)=c_{k-j}^{e o}(t)$, we need to distinguish three types of correlations. The simplest display, which manifestly shows the underlying symmetries, is

$$
\left(\begin{array}{c}
c_{k-j}^{e e}(t) \\
c_{k-j}^{e o}(t) \\
c_{k-j}^{o o}(t)
\end{array}\right)=\left(\begin{array}{c}
\bar{\gamma} / \gamma_{o} \\
\bar{\gamma} / \alpha \\
\bar{\gamma} / \gamma_{e}
\end{array}\right)(k-j) \int_{0}^{2 t} \frac{d \tau}{\tau} e^{-\tau} I_{k-j}(\alpha \tau) .
$$

Note that the last factor is of exactly the same form as in the ordinary Ising chain coupled to a single thermal bath, the only difference being the geometric mean of the two $\gamma$ 's here plays the role of $\gamma=\tanh \left(2 J / k_{b} T\right)$. Before turning to the general case, let us remind the reader that Eqs. (5) and (9) give the time-dependent correlations only for a system with no initial magnetization and two-spin correlations (e.g., a random distribution). In particular, these forms, also used in the next sections, should not be confused with the more general cases considered in Appendix B.

\section{Generating function and general multispin correlations}

In this section, starting from our knowledge of $m_{j}(t)$ and $c_{k, j}(t)$, we compute the generating function of the KISC, following [15]. By construction, this generating function allows us to find all correlation functions, subject to arbitrary initial conditions. A few additional technical details are provided in Appendix A.

The generating function is defined via $\Psi(\{\eta\}, t) \equiv\left\langle\Pi_{j}(1\right.$ $\left.\left.+\eta_{j} \sigma_{j}\right)\right\rangle_{t}$, where the $\left\{\eta_{j}\right\}$ are standard Grassmann variables $[15,17]$. In the thermodynamic limit, $L \rightarrow \infty$, it simplifies to

$$
\begin{aligned}
\Psi(\{\eta\}, t)= & \left\langle\prod_{j}\left(1+\sigma_{j} \sum_{k} \eta_{k} M_{k, j}(t)\right)\right\rangle_{0} \\
& \times \exp \left(\sum_{j_{2}>j_{1}} \eta_{j_{1}} \eta_{j_{2}} c_{j_{2}, j_{1}}(t)\right) .
\end{aligned}
$$

If the initial magnetization and all initial correlations vanish, the average $\langle\cdots\rangle_{0}$ on the right-hand side of Eq. (10) reduces to unity, and one recovers the bilinear form for $\Psi(\{\eta\}, t)$ which we already reported in [11]. Equation (10) is one of the key results of this paper. 
Given the generating function, all correlation functions can be obtained by simple differentiation $[11,15]$ : $\left\langle\sigma_{j_{1}} \cdots \sigma_{j_{n}}\right\rangle_{t}=\left[\partial^{n} \Psi(\{\eta\}, t)\right] /\left.\left(\partial \eta_{j_{n}} \cdots \partial \eta_{j_{1}}\right)\right|_{\{\eta\}=0}$. As an illustration, we compute the equal-time spin-spin correlation functions, for $k>j$,

$$
\begin{aligned}
\left\langle\sigma_{j} \sigma_{k}\right\rangle_{t}= & \left.\frac{\partial^{2} \Psi(\{\eta\}, t)}{\partial \eta_{k} \partial \eta_{j}}\right|_{\{\eta\}=0}=c_{k, j}(t) \\
& +\sum_{\ell<m}\left\langle\sigma_{\ell} \sigma_{m}\right\rangle_{0}\left[M_{\ell, j}(t) M_{m, k}(t)-M_{\ell, k}(t) M_{m, j}(t)\right] .
\end{aligned}
$$

We emphasize that this is a completely general result, valid for any initial conditions, whether homogeneous or inhomogeneous, translationally invariant or not. The two terms in Eq. (11) have simple interpretations. While the second term reflects the decay of the initial correlations, the first provides the buildup to the final stationary values given above Eq. (7). Thus, we see explicitly how the stationary spin-spin correlation function becomes independent of the initial values.

Higher-order correlations can also be evaluated but are rather complex for general initial conditions. For uncorrelated, nonmagnetized initial conditions, however, they simplify significantly [11]. For example, the four-point function $\left\langle\sigma_{j_{1}} \sigma_{j_{2}} \sigma_{j_{3}} \sigma_{j_{4}}\right\rangle_{t}$ factorizes into two-point functions, according to $\left\langle\sigma_{j_{1}} \sigma_{j_{2}} \sigma_{j_{3}} \sigma_{j_{4}}\right\rangle_{t}=c_{j_{2}, j_{1}}(t) c_{j_{4}, j_{3}}(t)-c_{j_{3}, j_{1}}(t) c_{j_{4}, j_{2}}(t)$ $+c_{j_{4}, j_{1}}(t) c_{j_{3}, j_{2}}(t)$ for $j_{4} \geqslant j_{3} \geqslant j_{2} \geqslant j_{1}$ [11]. Similar factorizations hold for all correlations. Their steady-state behavior can be computed directly from the master equation [9] or from the stationary limit of the generating function, $\Psi(\{\eta\}, \infty)=\exp \left[\Sigma_{k>j} \eta_{j} \eta_{k} c_{k, j}(\infty)\right]$. Thanks to this simple form, the $2 n$-point correlations factorize into a product of two-point correlations: $\left\langle\sigma_{j_{1}} \sigma_{j_{2}} \cdots \sigma_{j_{2 n-1}} \sigma_{j_{2 n}}\right\rangle_{\infty}$ $=\left\langle\sigma_{j_{1}} \sigma_{j_{2}}\right\rangle_{\infty} \cdots\left\langle\sigma_{j_{2 n-1}} \sigma_{j_{2 n}}\right\rangle_{\infty}$, where $j_{2 n}>j_{2 n-1}>\cdots>j_{2}>j_{1}$.

Finally, following Refs. [5,11], we can also derive the unequal-time spin-spin correlation functions $c_{k, j}\left(t^{\prime} ; t\right)$ describing how a spin on site $k$ at time $t$ is correlated with the spin on site $j$ at a later time $t+t^{\prime}$,

$$
\begin{aligned}
c_{k, j}\left(t^{\prime} ; t\right)= & \sum_{\ell} M_{j \ell}\left(t^{\prime}\right)\left\langle\sigma_{k} \sigma_{\ell}\right\rangle_{t} \\
= & \sum_{\ell} M_{j, \ell}\left(t^{\prime}\right) c_{k, \ell}(t)+\sum_{\ell} \sum_{k_{1}<\ell_{1}}\left\langle\sigma_{k_{1}} \sigma_{\ell_{1}}\right\rangle_{0} M_{j, \ell}\left(t^{\prime}\right) \\
& \times\left[M_{k_{1}, k}(t) M_{\ell_{1}, \ell}(t)-M_{k_{1}, \ell}(t) M_{\ell_{1}, k}(t)\right] .
\end{aligned}
$$

As an illustration of these general results, in Appendix B we specifically compute the spin-spin correlation functions for general translationally invariant initial conditions.

\section{CONSEQUENCES FOR A REACTION-DIFFUSION MODEL WITH ALTERNATING RATES}

In this section, our exact results will be translated into the language of the corresponding reaction-diffusion model. We first associate a site $\hat{j}$ on the dual lattice with every bond $(j$ $-1, j$ ) of the original chain. Since the particles of the RDS are identified with domain walls in the spin chain, they ob- viously reside on the dual lattice. Each site $\hat{j}$ can be occupied by at most one particle, described by an occupation variable $n_{\hat{j}}$ which takes the value 0 (1) if the site is empty (occupied). Since a domain wall involves two neighboring spins, the mapping from spin to particle language is nonlinear, namely, $n_{j}=\frac{1}{2}\left[1-\sigma_{j-1} \sigma_{j}\right]$. As before, we seek the probability, $\hat{P}(\{n\}, t)$, to find configuration $\{n\}$ at time $t$, and its averages: the local particle density $\rho_{j}^{\hat{j}}(t) \equiv\left\langle n_{j}\right\rangle_{t} \equiv \sum_{\{n\}} n_{j} \hat{P}(\{n\}, t)$ and the $m$-point correlation functions, $\left\langle n_{\hat{j}_{1}} \cdots n_{\hat{j}_{m}}\right\rangle_{t}$ $\equiv \sum_{\{n\}} n_{\hat{j}_{1}} \cdots n_{j_{m}} \hat{P}(\{n\}, t)$. To simplify notation, we continue to denote averages by $\langle\cdot\rangle_{t}$ for both spins and occupation variables, even though they are controlled by different statistical weights, $P(\{\sigma\}, t)$ and $\hat{P}(\{n\}, t)$, respectively. In each case, it should be perfectly clear from the context which distribution is relevant. The dynamics of our model is characterized by symmetric diffusion of particles (with rate $1 / 2$ and pair annihilation/creation of particles with spatially alternating rates $\left(1 \pm \gamma_{j}\right) / 2$. In this case, the two particles are created on the (dual lattice) sites $\hat{j}$ and $\hat{j}+1$, by flipping a spin on the (original lattice) site $j$. Since $\gamma_{j}$ can be positive or negative, subject only to $-1 \leqslant \gamma_{j} \leqslant 1$ for all $j$, two very distinct behaviors emerge: (i) when both $\gamma_{e}$ and $\gamma_{o}$ are positive (corresponding to positive "temperatures" in the spin model), the annihilation process always occurs with a larger rate than the creation process, irrespective of whether $j$ is even or odd; (ii) when, e.g., $\gamma_{o}$ is negative and $\gamma_{e}$ positive, the system displays a mild site-dependent frustration: at even sites $j$ (i.e., $\hat{j}$ even and $\hat{j}+1$ odd), annihilation is more likely than creation, whereas the situation is reversed on odd sites (with $\hat{j}$ odd and $\hat{j}+1$ even). As we will see shortly, this gives rise to oscillatory dynamics.

Before diving into the details, some further remarks on physical realizations of this model are in order. When the rates are uniform $\left(\gamma_{e}=\gamma_{o}\right)$, it is well known that such an RDS describes the dynamics of photoexcited solitons in conjugated polymers or linear chain compounds. MX chain compounds, $\left[\operatorname{Pt}(e n)_{2}\right]\left[\operatorname{Pt}(e n) \mathrm{Cl}_{2}\right] Y_{4}$, where $Y$ stands for $\mathrm{ClO}_{4}$ or $\mathrm{BF}_{4}$ and $(e n)$ for enthylenediamine, are of particular experimental interest $[12,13]$. In these compounds, photogenerated solitons are so long-lived that they can be experimentally studied. Irradiation with continuous-wave (nonpulsed) blue light generates soliton-antisoliton pairs which can diffuse apart or annihilate. Their static and dynamic properties are in quantitative agreement with theoretical models [4,18]. Since creation, annihilation, and hopping rates can be controlled by tuning the laser power, we believe that spatially alternating rates such as ours will be generated if an MX chain compound is exposed to a spatially modulated light intensity.

Returning to our model, our goal in this section is first to derive all correlation functions from our exact solution of the KISC. We will also comment on the validity of a simple mean-field theory which is widely used for the homogeneous $\left(\gamma_{e}=\gamma_{o}\right)$ case $[18,19]$. Further, we show that particle hops in the RDS develop a peculiar directional preference in the steady state, even though there is no explicit bias in the rates, boundary, or initial conditions. Finally, we illustrate how oscillatory behaviors may result from a competition of the underlying processes. 


\section{A. Density of particles in the RDS}

The observable of most immediate interest is the average density of particles, $\rho_{j}^{\wedge}(t)$, in the RDS. Its equation of motion can be derived easily from the associated master equation, resulting in

$$
\begin{aligned}
2 \frac{d}{d t} \rho_{j}^{\hat{j}}(t)= & \left(2-\gamma_{j}-\gamma_{j-1}\right)+\left[\gamma_{j-1} \rho_{j-1}(t)+\gamma_{j} \rho_{j+1}^{\hat{j}}(t)\right] \\
& -\left(4-\gamma_{j}-\gamma_{j-1}\right) \rho_{j}^{\hat{j}}(t) \\
& -2\left[\gamma_{j}\left\langle n_{j} n_{j-1}\right\rangle_{t}+\gamma_{j+1}\left\langle n_{j} n_{\hat{j}-1}\right\rangle_{t}\right] .
\end{aligned}
$$

It is worthwhile noting that this equation is the first member of an infinite hierarchy, connecting lower-order correlations to higher-order ones. In general, such hierarchies cannot be solved directly, without recourse to crude approximations. Here, the mapping to the spin chain develops its full power, allowing us to compute all correlation functions for the RDS.

The mapping from spins to particles implies that $\rho_{j}^{\hat{j}}(t)$ $\equiv\left\langle n_{j}\right\rangle=\frac{1}{2}\left[1-\left\langle\sigma_{j-1} \sigma_{j}\right\rangle_{t}\right]$, so that we can just turn to Eq. (11) to read off the answer. To express it fully in RDS language, we also need to translate the initial correlations, $\left\langle\sigma_{k} \sigma_{\ell}\right\rangle_{0}$. For $k<\ell$ and any $t$ (including $t=0$ ), we may write $\quad\left\langle\sigma_{k} \sigma_{\ell}\right\rangle_{t}=\left\langle\sigma_{k} \sigma_{k+1} \sigma_{k+1} \sigma_{k+2} \cdots \sigma_{\ell-1} \sigma_{\ell}\right\rangle_{t}=\left\langle\left(1-2 n_{k+1}\right)(1\right.$ $\left.\left.-2 n_{\hat{k}+2}\right) \cdots\left(1-2 n_{\ell}\right)\right\rangle_{t}[18,20]$ when we obtain, for arbitrary initial condition,

$$
\begin{aligned}
\rho_{j}^{\hat{j}}(t)= & \frac{1}{2}\left\{1-c_{j, j-1}(t)\right\}-\frac{1}{2} \sum_{\hat{k}<\hat{\ell}}\left\langle\left(1-2 n_{\hat{k}+1}\right)\right. \\
& \left.\times\left(1-2 n_{\hat{k}+2}\right) \cdots\left(1-2 n_{\hat{\ell}}\right)\right\rangle_{0} \\
& \times\left[M_{k, j-1}(t) M_{\ell, j}(t)-M_{k, j}(t) M_{\ell, j-1}(t)\right] .
\end{aligned}
$$

Since the "propagators" $M_{i, j}(t)$ decay exponentially as $t$ $\rightarrow \infty$, the steady-state density is independent of initial conditions and spatially uniform,

$$
\rho(\infty) \equiv \rho_{j}(\infty)=\frac{1}{2}\left(1-\frac{\bar{\gamma}}{\sqrt{\gamma_{e} \gamma_{o}}} \omega\right) .
$$

In Appendix B, we explicitly evaluate Eq. (14) for a generic but simple initial condition, characterized by a uniform, uncorrelated initial distribution of particles, with density $\rho(0)$. For simplicity, we discuss only its long-time limit here, for $\rho(0)=1 / 2$. We observe two distinct kinds of behaviors.

(i) When $\gamma_{e} \gamma_{o}>0$, the stationary density of particles is approached exponentially fast [except when $\gamma_{e}=\gamma_{o}= \pm 1$, see Eq. (B15)], with inverse relaxation time $2(1-\alpha)$, and a subdominant power-law prefactor $t^{-3 / 2}$,

$$
\rho(t)=\frac{1}{2}\left(1-\frac{\bar{\gamma}}{\alpha} \int_{0}^{2 t} \frac{d \tau}{\tau} e^{-\tau} I_{1}(\alpha \tau)\right) \simeq \rho(\infty)+\frac{t^{-3 / 2} e^{-2(1-\alpha) t}}{2 \sqrt{2 \pi \alpha}(1-\alpha)} .
$$

This long-time behavior is very similar to that found in the usual $\left(\gamma_{e}=\gamma_{o} \neq \pm 1\right)$ pair diffusion, annihilation, and creation process $A A \rightleftarrows \varnothing \varnothing[4,18]$.

(ii) For $\gamma_{o} \gamma_{e}<0$, we observe a competition between the different processes. For example, when $-1 \leqslant \gamma_{o}<0$ and 0 $<\gamma_{e} \leqslant 1$, the annihilation (creation) reaction dominates on even (odd) sites. As a result, the stationary density is reached exponentially fast with damped oscillations,

$$
\begin{aligned}
\rho(t) & =\frac{1}{2}\left(1-\frac{\bar{\gamma}}{|\alpha|} \int_{0}^{2 t} \frac{d \tau}{\tau} e^{-\tau} J_{1}(\alpha \tau)\right) \\
& \simeq \rho(\infty)-e^{-2 t}\left[\frac{\sin \left(2|\alpha| t-\frac{\pi}{4}\right)+|\alpha| \cos \left(2|\alpha| t-\frac{\pi}{4}\right)}{4\left(1+|\alpha|^{2}\right) t \sqrt{\pi|\alpha| t}}\right] .
\end{aligned}
$$

For initial densities other than $1 / 2$, as shown in Appendix $\mathrm{B}$, only the amplitude, or the subdominant power-law prefactor, of the expressions (16) and (17) changes. Since they depend on all parameters of the model, including the initial density, the dynamics is manifestly nonuniversal.

\section{B. Two-point correlation functions of the RDS}

A deeper understanding of the time-dependent spatial structures of our RDS is provided by the $m$-point correlation functions, $\left\langle n_{\hat{j}_{1}} \cdots n_{\hat{j}_{m}}\right\rangle_{t}$, of such a model. These are related to the correlation functions of the dual spin chain, via $\left\langle n_{\hat{j}_{1}} \cdots n_{\hat{j}_{m}}\right\rangle_{t}=2^{-m}\left\langle\left(1-\sigma_{j_{1}-1} \sigma_{j_{1}}\right) \cdots\left(1-\sigma_{j_{m}-1} \sigma_{j_{m}}\right)\right\rangle_{t}$, and are therefore exactly known. It is interesting to note that the $m$-point correlation function for the RDS is a superposition of all $2 n$-point correlation functions for the spin chain, with $n=1,2, \cdots, m$. In the following, we focus on the most directly observable correlation, namely, the two-point function. To avoid unnecessary technical complications which add little insight, we specifically consider a system that is initially homogeneously half-filled with $A$ particles, without any initial correlations: $\rho_{j}^{\hat{\gamma}}(0)=1 / 2$ and $\left\langle n_{j}(0) n_{\hat{k}}(0)\right\rangle=1 / 4$ for $\hat{j} \neq \hat{k}$. Such an initial configuration corresponds, in the KISC picture, to a system with initially neither magnetization nor correlations. In this case, as we showed in [11], the generating function takes a rather simple bilinear form which simplifies the spin-spin correlations.

With this initial condition, both the spin chain and the RDS are translationally invariant, modulo period 2 . As a result, the two-point correlations $\mathcal{C}_{\hat{k}-\hat{j}}^{\mu(\hat{k}), \mu(\hat{j})}(t) \equiv\left\langle n_{j} n_{\hat{k}}\right\rangle_{t}$ between two sites $\hat{j}$ and $\hat{k}$ (with $\hat{k}>\hat{j}$ ) depend only on the distance $\hat{k}$ $-\hat{j}$ and the parity $\mu(\hat{k}), \mu(\hat{j}) \in\{e, o\}$ of the two sites. We therefore need to distinguish four distinct correlation functions: $\mathcal{C}_{\hat{k}-\hat{j}}^{e e}(t), \mathcal{C}_{\hat{k}-\hat{j}}^{e o}(t), \mathcal{C}_{\hat{k}-\hat{j}}^{o e}(t)$, and $\mathcal{C}_{\hat{k}-\hat{j}}^{o o}(t)$. By virtue of our mapping to the KISC, these are determined by the two- and four-point spin correlations as explained in Sec. III C [from Eqs. (9) and (10)]. Exploiting translational invariance, the two-point correlations for the RDS, for $\hat{k}>\hat{j}$, then follow as

$$
\left\langle n_{j} n_{k}\right\rangle_{t}=\frac{1}{4}\left\{\left[1-c_{1}^{e o}(t)\right]^{2}-\left\langle\sigma_{j} \sigma_{k}\right\rangle_{t}^{2}\right\}+\frac{1}{4}\left\langle\sigma_{j-1} \sigma_{k}\right\rangle_{t}\left\langle\sigma_{j} \sigma_{k-1}\right\rangle_{t} .
$$

Now we are ready to discuss our results. First of all, we consider a special case, namely, nearest-neighbor correlations. If $\hat{k}=\hat{j}+1$, Eq. (18) reduces to 


$$
\left\langle n_{j} n_{\hat{j}+1}\right\rangle_{t}= \begin{cases}\frac{1-2 c_{1}^{e o}(t)+c_{2}^{o o}(t)}{4}=\mathcal{C}_{1}^{o e}(t), & \hat{j} \text { even } \\ \frac{1-2 c_{1}^{e o}(t)+c_{2}^{e e}(t)}{4}=\mathcal{C}_{1}^{e o}(t), & \hat{j} \text { odd } .\end{cases}
$$$$
\mathcal{C}_{1}^{o e}(\infty)=\rho^{2}(\infty)-\left(\gamma_{e}^{2}-\gamma_{o}^{2}\right)\left(\frac{\omega}{4 \alpha}\right)^{2}
$$

and

$$
\mathcal{C}_{1}^{e o}(\infty)=\rho^{2}(\infty)+\left(\gamma_{e}^{2}-\gamma_{o}^{2}\right)\left(\frac{\omega}{4 \alpha}\right)^{2} .
$$

Again, we should emphasize that the quantities $c_{n}^{e o}(t)$, $c_{n}^{e e}(t)$, and $c_{n}^{o o}(t)$ which appear in this section are the spin correlations for a particular initial condition [cf. Eq. (9)], in contrast to the more general correlations computed in Appendix B.

It is interesting to note that, generically, $\mathcal{C}_{1}^{o e}(t) \neq \mathcal{C}_{1}^{e o}(t)$. Of course, after a little thought this becomes less surprising, since $\left\langle n_{j} n_{\hat{j}+1}\right\rangle_{t}$ involves the four-spin correlation $\left\langle\sigma_{j-1} \sigma_{j} \sigma_{j} \sigma_{j+1}\right\rangle_{t}=\left\langle\sigma_{j-1} \sigma_{j+1}\right\rangle_{t}$. So, if $\hat{j}$ is odd (even), both $j$ -1 and $j+1$ are even (odd), leading to a contribution of $c_{2}^{e e}(t)$ versus $c_{2}^{o o}(t)$, respectively.

For the general case, when $\hat{k}$ and $\hat{j}$ are not nearest neighbors, this difference between $\mathcal{C}_{\hat{k}-j}^{e o}(t)$ and $\mathcal{C}_{\hat{k}-\hat{j}}^{o e}(t)$ does not persist. If $\hat{k}$ is even and $\hat{j}$ is odd, we find

$$
\mathcal{C}_{\hat{k}-j}^{e o}(t)=\frac{1}{4}\left\{\left[1-c_{1}^{e o}(t)\right]^{2}-\left[c_{k-j}^{e o}(t)\right]^{2}\right\}+\frac{1}{4} c_{k-j+1}^{e e}(t) c_{k-j-1}^{o o}(t),
$$

and for $\hat{k}$ odd and $\hat{j}$ even one obtains

$$
\mathcal{C}_{\hat{k}-\hat{j}}^{o e}(t)=\frac{1}{4}\left\{\left[1-c_{1}^{e o}(t)\right]^{2}-\left[c_{k-j}^{e o}(t)\right]^{2}\right\}+\frac{1}{4} c_{k-j+1}^{o o}(t) c_{k-j-1}^{e e}(t) .
$$

Thanks to the simple relation between even-even and oddodd spin correlations, Eq. (9), the two right-hand sides are now identical.

A similar line of reasoning shows that $\mathcal{C}_{\hat{k}-\hat{j}}^{e e}(t)=\mathcal{C}_{\hat{k}-\hat{j}}^{o o}(t)$ for arbitrary separation $\hat{k}-\hat{j}$. Invoking the two-spin correlations again, we may write

$$
\begin{aligned}
\mathcal{C}_{\hat{k}-\hat{j}}^{e e}(t)= & \mathcal{C}_{\hat{k}-\hat{j}}^{o o}(t)=\frac{1}{4}\left\{\left[1-c_{1}^{e o}(t)\right]^{2}-c_{k-j}^{o o}(t) c_{k-j}^{e e}(t)\right. \\
& \left.+c_{k-j+1}^{e o}(t) c_{k-j-1}^{e o}(t)\right\} .
\end{aligned}
$$

In the following, we discuss the consequences of these results. We first consider the steady state. Recalling our previous analysis of the spin correlations, Eq. (7), the stationary limit of the density-density correlations becomes very simple: Provided $\hat{k}-\hat{j}>1$, we find $\mathcal{C}_{\hat{k}-\hat{j}}^{\mu(\hat{k}), \mu(\hat{j})}(\infty)=\frac{1}{4}[1$ $\left.-c_{1}^{e o}(\infty)\right]^{2}=\rho^{2}(\infty)$. In other words, the two-point correlations of non-nearest-neighbor sites factorize into one-point functions, independent of parity. This kind of mean-field-like behavior is typical of free-fermion systems $[4,18]$. However, the nonequilibrium nature of this model still imposes its signature. Turning to the nearest-neighbor correlations, we find that this simple factorization no longer holds-except in the special case where $\gamma_{e}=\gamma_{o}$. More specifically, we find
Considering, e.g., $0<\gamma_{o}<\gamma_{e}$, we find that $\mathcal{C}_{1}^{e o}(\infty)$ is enhanced over the mean-field result while $\mathcal{C}_{1}^{o e}(\infty)$ is suppressed. This can be understood easily: Since $\gamma_{o}<\gamma_{e}$ implies $T_{e}$ $<T_{o}$, energetically costly spin flips occur more frequently on odd sites $j$, creating a particle pair on the nearest-neighbor dual sites $(\hat{j}+1, \hat{j})$. Clearly, these sites form an $(e, o)$ pair. Moreover, the rate for pair annihilation is lower on $(e, o)$ sites. Hence, particle pairs are more likely to reside on $(e, o)$ than on $(o, e)$ sites. This also implies that $(e, o)$ sites act as net particle sources, while $(o, e)$ sites function as sinks [10]. Not surprisingly, therefore, we find $\mathcal{C}_{1}^{e o}(\infty)>\mathcal{C}_{1}^{o e}(\infty)$. By virtue of this reasoning, it is also immediately apparent that this difference can only persist for nearest-neighbor correlations. The same argument holds for $\gamma_{o}<0<\gamma_{e}$.

A direct consequence of $\mathcal{C}_{1}^{o e}(t) \neq \mathcal{C}_{1}^{e o}(t)$ is the presence of a peculiar directional preference in the RDS. If we consider a particle on site $\hat{j}$, we can ask for the average rate, $\mathcal{R}_{\hat{j}}(t)$, with which it will jump to the left (i.e., to site $\hat{j}-1)$ versus to the right, defined as $\mathcal{R}_{\hat{j}}(t) \equiv \frac{1}{2}\left\langle n_{\hat{j}}\left(1-n_{\hat{j}+1}\right)-n_{j}\left(1-n_{\hat{j}-1}\right)\right\rangle_{t}$. Here, the first (second) term is the average rate for a particle on site $\hat{j}$ to jump to site $\hat{j}+1(\hat{j}-1)$. In our case, one might expect this difference to vanish since neither bulk rates nor boundaries impose a directional bias. Moreover, to avoid a potential bias at $t=0$, we start from a translationally invariant initial condition with $\rho(0)=1 / 2$. Yet, since $\mathcal{R}_{j}(t) \propto \mathcal{C}_{1}^{o e}(t)-\mathcal{C}_{1}^{e o}(t)$, it is manifestly nonzero. Explicitly, we find

$$
\mathcal{R}_{\hat{j}}(t)= \begin{cases}\frac{1}{8}\left[1-\frac{\gamma_{o}}{\gamma_{e}}\right] c_{2}^{e e}(t), & \hat{j} \text { even } \\ \frac{1}{8}\left[\frac{\gamma_{o}}{\gamma_{e}}-1\right] c_{2}^{e e}(t), & \hat{j} \text { odd },\end{cases}
$$

which even persists in the steady state,

$$
\mathcal{R}_{j}(\infty)= \begin{cases}\left(\gamma_{e}^{2}-\gamma_{o}^{2}\right)\left(\frac{\omega}{4 \alpha}\right)^{2}, & \hat{j} \text { even } \\ \left(\gamma_{o}^{2}-\gamma_{e}^{2}\right)\left(\frac{\omega}{4 \alpha}\right)^{2}, & \hat{j} \text { odd } .\end{cases}
$$

Specifically, for $\gamma_{o}<\gamma_{e}$, particles on an even (odd) site jump preferentially to the right (left). Of course, this directional preference vanishes as soon as $\gamma_{e}=\gamma_{o}$. Moreover, even when it is nonzero, it does not generate a mass current. Counting the net flow of particles between sites $\hat{j}$ and $\hat{j}+1$, the natural definition of such a current is $\mathcal{J}_{\hat{j}}(t)=\frac{1}{2}\left\langle\left(1-n_{j}\right) n_{\hat{j}+1}-n_{j}(1\right.$ $\left.\left.-n_{\hat{j}+1}\right)\right\rangle_{t}$. Clearly, this expression reduces to a density difference which vanishes for all times provided the initial condition is homogeneous. For inhomogeneous initial condition, $\mathcal{J}_{\hat{j}}(t)$ exhibits nonzero transients for finite times but decays as $t \rightarrow \infty$.

Let us conclude this section with a few brief remarks about the validity of the mean-field approximation for this system. We already noted that it does not predict the nearestneighbor correlations correctly, except in the special case 
$\gamma_{e}=\gamma_{o}$. We now show that it also generically misses the stationary density.

We begin by recalling Eq. (13). Seeking a translationally invariant (modulo 2) solution with $\rho_{2 j} \hat{j}(t)=\rho_{e}(t), \rho_{2 \hat{j}+1}(t)$ $=\rho_{o}(t)$ for all $\hat{j}$, the mean-field approximation corresponds to truncating two-point functions: $\left\langle n_{j}(t) n_{\hat{j}-1}(t)\right\rangle \simeq \rho_{e}(t) \rho_{o}(t)$. Starting from a uniform initial density $\rho(0)$, we find $\rho_{e}(t)$ $=\rho_{o}(t) \equiv \rho_{\mathrm{MF}}(t)$, with

$$
\begin{aligned}
\rho_{\mathrm{MF}}(t) & =\frac{\rho_{p}\left[\rho(0)-\rho_{m}\right]-\rho_{m}\left[\rho(0)-\rho_{p}\right] e^{-t \sqrt{4-\left(\gamma_{e}+\gamma_{o}\right)^{2}}}}{\rho(0)-\rho_{m}+\left[\rho(0)-\rho_{p}\right] e^{-t \sqrt{4-\left(\gamma_{e}+\gamma_{o}\right)^{2}}}} \\
& \simeq \rho_{p}-\rho_{m}\left(\frac{\rho(0)-\rho_{p}}{\rho(0)-\rho_{m}}\right) e^{-t \sqrt{4-\left(\gamma_{e}+\gamma_{o}\right)^{2}}},
\end{aligned}
$$

where

$$
\rho_{p, m}=\frac{1}{2}\left[1-\frac{2}{\gamma_{e}+\gamma_{o}} \pm \frac{\sqrt{4-\left(\gamma_{e}+\gamma_{o}\right)^{2}}}{\gamma_{e}+\gamma_{o}}\right] .
$$

The stationary limit is clearly $\rho_{\mathrm{MF}}(\infty)=\rho_{p}$ which differs from our exact result, Eq. (15), except if $\gamma_{e}=\gamma_{o}$. In other words, the remarkable accuracy $[18,19]$ of the mean-field approximation for the stationary state of the uniform system $\left(\gamma_{e}\right.$ $=\gamma_{o}$ ) appears to be an "accident" due to the fact that when rates are uniform, the steady state is a product measure. We also note that the exact relaxation time to the steady state, $\tau_{\text {exact }}=\left[2-\sqrt{\gamma_{e} \gamma_{o}}\right]^{-1}$, does not coincide with the mean-field prediction, $\tau_{\mathrm{MF}}=\left[\sqrt{4-\left(\gamma_{e}+\gamma_{o}\right)^{2}}\right]^{-1}$. For such dynamic quantities, the exact and the approximate results differ for any choice of $\gamma_{e}$ and $\gamma_{o}$. In particular, the mean-field theory always predicts an exponential decay to the steady state, completely missing the possibility of oscillatory behavior.

\section{SOLVABILITY AND RELATIONSHIP WITH FREE FERMION SYSTEMS}

The crucial ingredient for the solvability of the KISC is the quadratic spin dependence of its Glauber-like kinetics. Thanks to this simple form, the hierarchy of equations for the correlation functions is closed: to solve the equations for the $N$-spin correlation functions, one needs to know only $m$-point correlations with $m \leqslant n$.

In RDS language, the dynamics of the particles can be rewritten as a free fermion model, by defining a suitable quadratic (but non-Hermitian) "stochastic Hamiltonian." Following standard methods $[3,4,18,21]$, we can rewrite the master equation for the RDS as a formal imaginary-time Schrödinger equation: $(d / d t)|P(t)\rangle=-H|P(t)\rangle$. The Hamiltonian $H$ is constructed by associating the usual Pauli matrices $\sigma_{\hat{j}}^{-}\left(\sigma_{\hat{j}}^{+}\right)$with the creation (annihilation) of a particle at site $\hat{j}$,

$$
\begin{aligned}
-2 H= & \sum_{\hat{j} \text { even }}\left[\sigma_{\hat{j}}^{+} \sigma_{\hat{j}+1}^{-}+\sigma_{\hat{j}}^{-} \sigma_{\hat{j}+1}^{+}+\left(1+\gamma_{e}\right) \sigma_{\hat{j}}^{+} \sigma_{\hat{j}+1}^{+}\right. \\
& +\left(1-\gamma_{e}\right) \sigma_{\hat{j}}^{-} \sigma_{\hat{j}+1}^{-}-\gamma_{e}\left(\sigma_{\hat{j}}^{-} \sigma_{\hat{j}}^{+}+\sigma_{\hat{j}+1}^{-} \sigma_{\hat{j}+1}^{+}\right) \\
& \left.-\left(1-\gamma_{e}\right)\right]+\sum_{\hat{j} \text { odd }}\left[\sigma_{\hat{j}}^{+} \sigma_{\hat{j}+1}^{-}+\sigma_{\hat{j}}^{-} \sigma_{\hat{j}+1}^{+}\right.
\end{aligned}
$$

$$
\begin{aligned}
& +\left(1+\gamma_{o}\right) \sigma_{\hat{j}}^{+} \sigma_{\hat{j}+1}^{+}+\left(1-\gamma_{o}\right) \sigma_{\hat{j}}^{-} \sigma_{\hat{j}+1}^{-} \\
& \left.-\gamma_{o}\left(\sigma_{\hat{j}}^{-} \sigma_{\hat{j}}^{+}+\sigma_{\hat{j}+1}^{-} \sigma_{\hat{j}+1}^{+}\right)-\left(1-\gamma_{o}\right)\right] .
\end{aligned}
$$

The key to the solvability of this Schrödinger equation lies in the bilinear dependence of the Hamiltonian on the Pauli matrices. This is due to the fact that the spin-flip rates (1) implicitly fulfill the free-fermion constraint $[3,4,18]$. In RDS language, this condition requires that the sum of the particle diffusion rates equal the sum of the (local) annihilation and creation rates, i.e., $1 / 2+1 / 2=\left(1+\gamma_{j}\right) / 2+\left(1-\gamma_{j}\right) / 2$ with $j \in\{e, o\}$ in our case. If this relation is violated, $H$ includes quartic terms, of the form $\sum_{\hat{j}} \sigma_{\hat{j}}^{-} \sigma_{\hat{j}}^{+} \sigma_{\hat{j}+1}^{-} \sigma_{\hat{j}+1}^{+}$, and the associated RDS can no longer be solved exactly. It can, of course, be simulated, and for those cases investigated so far, it appears that the quartic terms are irrelevant for the longtime dynamics $[4,19]$. It is also worth noting that the freefermion constraint is not particularly artificial: the simplest models for photogenerated solitons in MX chain compounds satisfy it quite naturally [12].

Here we decided to invoke generating function techniques instead of diagonalizing Eq. (27). In our view, this is the most convenient and systematic approach to solve simultaneously both the KISC and RDS, for two reasons. First, the free-fermion approach requires various technical steps (e.g., introduction of so-called pseudofermion operators and a Bogoliubov-like transformation) which make the general treatment rather involved, especially for arbitrary initial conditions $[4,18]$. Further, the diagonalization of Eq. (27) yields only correlation functions with an even number of spins (see Secs. III and IV); the calculation of correlations involving an odd number of spins requires a dual transformation of Eq. (27) into a new stochastic Hamiltonian which must also be diagonalized [20].

Let us also mention that damped oscillatory decay has been observed before in certain reaction-diffusion models [3]. However, those models, and hence the physical mechanisms leading to the oscillations, are completely different from ours. As an example, a diffusion-limited fusion model [3] is defined by three processes: (i) biased diffusion: $A \varnothing$ $\rightarrow \varnothing A$ with rate $D(1+\eta), \varnothing A \rightarrow A \varnothing$ with rate $D(1-\eta)$ (with $0<\eta \leqslant D$ ); (ii) biased fusion: $A A \rightarrow \varnothing A$ with rate $D(1+2 \eta)$, $A A \rightarrow A \varnothing$ with rate $D(1-2 \eta)$; and (iii) homogeneous pair production: $\varnothing \varnothing \rightarrow A A$, with rate $D$. With this special choice of rates, the equation of motion for the density closes and becomes solvable. In order to observe oscillatory decay of the particle density, the initial condition must be inhomogeneous. For a homogeneous initial condition, the density decays exponentially. In contrast, the equation in our reactiondiffusion model does not close, and the oscillatory behavior is generic: it occurs for any initial condition, inhomogeneous or not.

\section{CONCLUSIONS}

To summarize, we have presented a full exact solution for the dynamics of a nonequilibrium Ising spin chain, with arbitrary initial condition. The model is characterized by a gen- 
eralization of Glauber dynamics: spins on even/odd sites are coupled to alternating temperatures, $T_{e}$ and $T_{o}$. We obtain all correlation functions from a generating functional. As an illustration, we have discussed the equal-time and the twotime spin-spin correlation functions.

Identifying domain walls in the spin system with particles on the dual lattice, the model can also be interpreted as a reaction-diffusion system. Particles are created and annihilated in pairs; the rates for these processes alternate from even to odd sites. This mapping opens up an interesting extension of parameter space: while negative temperatures are unphysical for the spin chain, the corresponding rates are perfectly natural in the context of the RDS. By expressing particle-particle correlations as superpositions of spin-spin correlation functions, the RDS becomes exactly soluble. This is not entirely trivial since the BBGKY [22] hierarchy for the RDS is not closed: its solution is far from obvious unless one recognizes the connection to the spin chain.

For $0<\gamma_{e} \gamma_{o}$, energetically favorable spin flips always dominate over unfavorable ones, irrespective of whether they occur on even or odd sites. In RDS language, pair annihilation is always more probable than pair creation. As a consequence, we find that all quantities decay exponentially to their steady-state values. In contrast, for $\gamma_{e} \gamma_{o}<0$, we observe (damped) oscillatory behavior. Its origin can be traced to a competition of pair creation and annihilation on even versus odd sites on the original lattice: If, say, $\gamma_{o}<0$, then pair creation dominates over annihilation on odd sites while the relation is reversed on the even sites. Hence, a given initial particle density may first decrease, due to annihilation processes, and then recover, as the available empty sites are (partially) filled again by the strong creation process, and so on, until the stationary density is reached.

Remarkably, even in the absence of any bias in the rates, boundary, or initial conditions, particles still "know" the difference between right and left: For, e.g., $\gamma_{o}<\gamma_{e}$, particles on even (odd) sites jump preferentially to the right (left). Even though this directional preference does not lead to a systematic particle current, it is still somewhat surprising. However, once we recall that particles are most often created (annihilated) on pairs of neighboring sites, with the odd site on the left (right), we recognize that the directional preference is simply a response to this density gradient.

Since exact solutions, especially of a full nonequilibrium dynamics, are rare, we hope that our model can serve as a testing ground for various generalizations or approximations. The features reported here-exponential decays, damped oscillations, and directional preference-should be generic for a whole class of genuine out-of-equilibrium models. Moreover, they should be experimentally observable in MX chain compounds exposed to spatially modulated laser light.

\section{ACKNOWLEDGMENTS}

It is a pleasure to acknowledge fruitful discussions with I. Georgiev, H. Hilhorst, J.R. Heflin, and U.C. Täuber. M.M. acknowledges the support of the Swiss NSF Grant No. 81EL-68473. This work was also partially supported by U.S. NSF Grants No. DMR-008845, No. DMR-0308548, and No.
DMR-0414122.

\section{APPENDIX A: THE DERIVATION OF THE GENERATING FUNCTION}

In this appendix, we provide some details for the derivation of the generating function (10), which is one of the key results of this work. We follow Aliev's work and notation [15]. Aliev established that the generating functions of a very general class of disordered Glauber-Ising spin chains, including our case, can formally be expressed in terms of two functions $M_{j, k}^{ \pm}(t)$ and two additional quantities $W_{j, k}^{ \pm}(t)$, which depend in a very involved fashion on $M_{k, j}^{ \pm}(t)$. Below, we will see that these quantities are closely related to physical observables, namely, the magnetization and the two-point correlations. Here, we follow Aliev by noting that the Laplace transform of $\hat{M}_{j, k}^{ \pm}$is the inverse of an $L \times L$ band matrix $(s$ $+1) 1-\frac{1}{2} U^{ \pm}$. For our case, the entries of this matrix can be taken from the rates and read explicitly ( $L$ is even),

$$
\begin{gathered}
{\left[(s+1) 1-\frac{1}{2} U^{ \pm}\right]_{2 j-1, k}=(s+1) \delta_{2 j, k}-\frac{\gamma_{o}}{2}\left(\delta_{2 j-1, k-1}+\delta_{2 j-1, k+1}\right)} \\
(1<j \leqslant L / 2), \\
{\left[(s+1) 1-\frac{1}{2} U^{ \pm}\right]_{2 j, k}=(s+1) \delta_{j, k}-\frac{\gamma_{e}}{2}\left(\delta_{2 j, k-1}+\delta_{2 j, k+1}\right)} \\
\quad(1 \leqslant j<L), \\
{\left[(s+1) 1-\frac{1}{2} U^{ \pm}\right]_{1, k}=(s+1) \delta_{1, k}-\frac{\gamma_{o}}{2}\left(\delta_{2, k} \mp \delta_{k, L}\right),} \\
{\left[(s+1) 1-\frac{1}{2} U^{ \pm}\right]_{L, k}=(s+1) \delta_{L-1, k}-\frac{\gamma_{o}}{2}\left(\delta_{L-1, k} \mp \delta_{1, k}\right) .}
\end{gathered}
$$

Given Eqs. (A1), it is easy to evaluate the inverse of $\left[(s+1) 1-(1 / 2) U^{ \pm}\right]_{j, k}$,

$$
\hat{M}_{k, j}^{ \pm}=\frac{1}{L} \sqrt{\frac{\gamma_{k}}{\gamma_{j}}} \sum_{n=1}^{L} \frac{e^{i(k-j) \phi_{n}^{ \pm}}}{s+1-\alpha \cos \phi_{n}^{ \pm}},
$$

where $\phi_{n}^{+}=[\pi(2 n-1)] / L$ and $\phi_{n}^{-}=(2 \pi n) / L$, with $n$ $=1,2, \ldots, L$. In the thermodynamic limit $L \rightarrow \infty$, the two quantities $\hat{M}_{j, k}^{+}$and $\hat{M}_{j, k}^{-}$coincide whence we simply have

$$
\hat{M}_{k, j}^{ \pm} \rightarrow \hat{M}_{k, j}=\sqrt{\frac{\gamma_{k}}{\gamma_{j}}} \int_{0}^{2 \pi} \frac{d \phi}{2 \pi} \frac{e^{i(k-j) \phi}}{(s+1-\alpha \cos \phi)} .
$$

Taking the inverse Laplace transform of Eq. (A3), we recover Eq. (4) for the propagator.

Since the $W_{j, k}^{ \pm}(t)$ can be expressed in terms of the $M_{j, k}^{ \pm}(t)$, we may immediately conclude that $W_{j, k}^{ \pm}(t) \rightarrow W_{j, k}(t)$ as $L$ $\rightarrow \infty$. According to Aliev, $W_{j, k}(t)$ is simply the two-point correlation function, $\left\langle\sigma_{j} \sigma_{k \neq j}\right\rangle_{t}$, for a particular initial condition, namely, $m_{j}(0)=0$ and $\left\langle\sigma_{j} \sigma_{k \neq j}\right\rangle_{0}=0$. For our case, these correlations were given in Eq. (5). 
For readers familiar with Aliev's work [15], these remarks fill in the gaps between Aliev's formal and general analysis and the special case we are interested in here. It follows that the generating function of our KISC admits the compact and explicit representation of Eq. (10), which encodes the complete dynamics of the system.

\section{APPENDIX B: TRANSLATIONALLY INVARIANT INITIAL CONDITIONS: THE SPIN-SPIN CORRELATION FUNCTIONS AND THE PARTICLE DENSITY}

In Sec. III B, we have derived an exact expression, Eq. (11), for the spin-spin correlation functions of our KISC, valid for arbitrary initial conditions. Here, we impose a natural restriction, namely, translational invariance, on the initial conditions. Thanks to the symmetry, Eq. (11) simplifies considerably, as we will show now.

As we already pointed out in [11], for translationally invariant initial conditions, we only need to consider the correlations between spins at two even sites, two odd sites, and one even, one odd site. We denote these by $c_{2 n}^{e e}(t)$ $\equiv\left\langle\sigma_{2 \ell} \sigma_{2(\ell+n)}\right\rangle_{t}, \quad c_{2 n}^{o o}(t) \equiv\left\langle\sigma_{2 \ell-1} \sigma_{2 \ell-1+2 n}\right\rangle_{t}, \quad c_{2 n-1}^{e o}(t)=c_{2 n-1}^{o e}(t)$ $\equiv\left\langle\sigma_{2 \ell} \sigma_{2 \ell+2 n-1}\right\rangle_{t}=\left\langle\sigma_{2 \ell+1} \sigma_{2 \ell+2 n}\right\rangle_{t}$. Of course, there is no need to study $n<0$ cases. For the special case of zero initial magnetization and correlations, these correlations are already known [11] and are given by Eq. (9). Here, we seek their form in a more general case, starting from a homogeneous initial condition.

Let us recall from [11] that in the translationally invariant case, the quantities $a_{2 n}(t) \equiv(1 / 2)\left[\gamma_{e} c_{2 n}^{o o}(t)+\gamma_{o} c_{2 n}^{e e}(t)\right]$, $a_{2 n-1}(t) \equiv \alpha c_{2 n-1}^{e o}(t)$, obey the following simple equation: $(d / d t) a_{j}=-2 a_{j}+\alpha\left[a_{j-1}+a_{j+1}\right], j>0$ with the initial condition $a_{0}(t)=\bar{\gamma}$ and $\bar{\gamma} \equiv\left(\gamma_{e}+\gamma_{o}\right) / 2$. The equations of motion of the KISC [11] also give the following relationships among the correlators: $\quad c_{2 n}^{o o}(t)=\left(\gamma_{o} / \gamma_{e}\right) c_{2 n}^{e e}(t)+\left[c_{2 n}^{o o}(0)\right.$ $\left.-\left(\gamma_{o} / \gamma_{e}\right) c_{2 n}^{e e}(0)\right] e^{-2 t}$.

The explicit expressions for the correlators follow from Eq. (11), or by the methods of images directly from the expression $a_{n}(t)=a_{n}(\infty)+e^{-2 t} \Sigma_{m \geqslant 0}\left[a_{m}(0)-a_{m}(\infty)\right]$ $\times\left\{I_{n-m}(2 \alpha t)-I_{n+m}(2 \alpha t)\right\}$, where $a_{n}(\infty)=\bar{\gamma} \omega^{k}$ and $n \geqslant 0$. From the definitions of $a_{n}$, we immediately infer

$$
\begin{aligned}
c_{2 n}^{e e}(t)= & \frac{\gamma_{e}}{\gamma_{o}} c_{2 n}^{o o}(t)-\left(\frac{\gamma_{e}}{\gamma_{o}} c_{2 n}^{o o}(0)-c_{2 n}^{e e}(0)\right) e^{-2 t} \\
= & \frac{a_{2 n}(\infty)}{\gamma_{o}}+\frac{e^{-2 t}}{\gamma_{o}} \\
& \times \sum_{m \geqslant 0}\left[a_{2 m}(0)-a_{2 m}(\infty)\right]\left\{I_{2(n-m)}(2 \alpha t)-I_{2(n+m)}(2 \alpha t)\right\} \\
& +\frac{\alpha e^{-2 t}}{\gamma_{o}} \sum_{m>0}\left[c_{2 m-1}^{e o}(0)-c_{2 m-1}^{e o}(\infty)\right]\left\{I_{2(n-m)+1}(2 \alpha t)\right. \\
& \left.\left.-I_{2(n+m)-1}(2 \alpha t)\right\}-\frac{e^{-2 t}}{2 \gamma_{o}}\left[\gamma_{e} e_{2 n}^{o o}(0)-\gamma_{o} c_{2 n}^{e e}(0)\right] . \quad \text { (B } 1\right)
\end{aligned}
$$

Following the same steps for $c_{2 n-1}^{e o}=c_{2 n-1}^{o e}$, we obtain, for $(n$ $>0)$,

$$
\begin{aligned}
c_{2 n-1}^{e o}(t)= & c_{2 n-1}^{e o}(\infty)+e^{-2 t} \sum_{m>0}\left[c_{2 m-1}^{e o}(0)-c_{2 m-1}^{e o}(\infty)\right] \\
& \times\left\{I_{2(n-m)}(2 \alpha t)-I_{2(n+m-1)}(2 \alpha t)\right\}+\frac{e^{-2 t}}{\alpha} \sum_{m>0}\left[a_{2 m}(0)\right. \\
& \left.-a_{2 m}(\infty)\right]\left\{I_{2(n-m)-1}(2 \alpha t)-I_{2(n+m)-1}(2 \alpha t)\right\} .
\end{aligned}
$$

The expressions (B1) and (B2) illustrate that the time dependence of the spin-spin correlation function depends nontrivially on the initial condition, and we may therefore anticipate nonuniversal behavior. Of course, when $\gamma_{e}=\gamma_{o}$, the expressions (B1) and (B2) coincide with those obtained by Glauber [5].

An interesting situation occurs when, say, $\gamma_{o}$ is negative while $0<\gamma_{e} \leqslant 1$, so that $\alpha=i|\alpha|$. Then, we have $I_{2 n}(2 i|\alpha| t)$ $=(-1)^{n} J_{2 n}(2|\alpha| t)$ and $I_{2 n \pm 1}(2 i|\alpha| t)= \pm i(-1)^{n} J_{2 n \pm 1}(2|\alpha| t)$, where $J_{n}(x) \equiv \int_{0}^{\pi}(d q / \pi) \cos (x \sin q-n q)$ is the Bessel function of the first kind [16]. Further, when $\alpha=i|\alpha|$, the expressions (B1) and (B2) become

$$
\begin{aligned}
c_{2 n}^{e e}(t)= & \frac{\gamma_{e}}{\gamma_{o}} c_{2 n}^{o o}(t)-\left(\frac{\gamma_{e}}{\gamma_{o}} c_{2 n}^{o o}(0)-c_{2 n}^{e e}(0)\right) e^{-2 t} \\
= & -\frac{a_{2 n}(\infty)}{\left|\gamma_{o}\right|}-\frac{e^{-2 t}}{\left|\gamma_{o}\right|} \sum_{m \geqslant 0}\left[a_{2 m}(0)-a_{2 m}(\infty)\right](-1)^{n+m} \\
& \times\left\{J_{2(n-m)}(2|\alpha| t)-J_{2(n+m)}(2|\alpha| t)\right\}+\frac{|\alpha| e^{-2 t}}{\left|\gamma_{o}\right|} \\
& \times \sum_{m>0}\left[c_{2 m-1}^{e o}(0)-c_{2 m-1}^{e o}(\infty)\right](-1)^{n+m} \\
& \times\left\{J_{2(n-m)+1}(2|\alpha| t)+J_{2(n+m)-1}(2|\alpha| t)\right\} \\
& -\frac{e^{-2 t}}{2 \gamma_{o}}\left[\gamma_{e} c_{2 n}^{o o}(0)-\gamma_{o} c_{2 n}^{e e}(0)\right],
\end{aligned}
$$

$$
\begin{aligned}
c_{2 n-1}^{e o}(t)= & c_{2 n-1}^{e o}(\infty)+e^{-2 t} \sum_{m>0}\left[c_{2 m-1}^{e o}(0)-c_{2 m-1}^{e o}(\infty)\right](-1)^{(n+m)} \\
& \times\left\{J_{2(n-m)}(2|\alpha| t)+J_{2(n+m-1)}(2|\alpha| t)\right\} \\
& -\frac{e^{-2 t}}{|\alpha|} \sum_{m>0}\left[a_{2 m}(0)-a_{2 m}(\infty)\right](-1)^{(n+m)} \\
& \times\left\{J_{2(n+m)-1}(2|\alpha| t)-J_{2(n-m)-1}(2|\alpha| t)\right\} .
\end{aligned}
$$

In the long-time limit, these expressions exhibit a damped oscillatory approach to the stationary state.

We now turn to the equivalent RDS. As we have seen in Sec. IV A, the density of particles is related to the nearestneighbor spin correlations, $c_{1}^{e o}$, according to $\rho(t)=\frac{1}{2}[1$ $\left.-c_{1}^{e o}(t)\right]$. Here, our goal is to determine the long-time behavior of this density for a homogeneous (but otherwise arbitrary) initial concentration of particles $\rho(0)$. In this respect, the expressions (B2) and (B4) are not very practical as they involve infinite sums of Bessel functions. At this point, for further convenience, it is useful to introduce four auxiliary functions defined as follows (with $0 \leqslant \lambda \leqslant 1$ ): 


$$
\begin{gathered}
F_{1}(\lambda, t) \equiv \sum_{m>0} \lambda^{2 m-1} e^{-2 t}\left\{I_{2(m-1)}(2 \alpha t)-I_{2 m}(2 \alpha t)\right\} \\
=2 \lambda\left(1+\lambda^{2}\right) \int_{0}^{\pi} \frac{d q}{\pi} \frac{e^{-2 t(1-\alpha \cos q)} \sin ^{2} q}{1+\lambda^{4}-2 \lambda^{2} \cos 2 q}, \\
F_{2}(\lambda, t) \equiv \sum_{m>0} \lambda^{2 m} e^{-2 t}\left\{I_{2 m-1}(2 \alpha t)-I_{2 m+1}(2 \alpha t)\right\} \\
=2 \lambda^{2} \int_{0}^{\pi} \frac{d q}{\pi} e^{-2 t(1-\alpha \cos q)}\left[\frac{\sin 2 q \sin q}{1+\lambda^{4}-2 \lambda^{2} \cos 2 q}\right],
\end{gathered}
$$

$$
\begin{aligned}
G_{1}(\lambda, t) \equiv & -\sum_{m>0} \lambda^{2 m-1}(-1)^{m} e^{-2 t}\left\{J_{2(1-m)}(2|\alpha| t)+J_{2 m}(2|\alpha| t)\right\} \\
= & 2 \lambda\left(1+\lambda^{2}\right) \int_{0}^{\pi} \frac{d q}{\pi} e^{-2 t} \cos (2|\alpha| t \sin q) \\
& \times\left[\frac{\cos ^{2} q}{1+\lambda^{4}+2 \lambda^{2} \cos 2 q}\right],
\end{aligned}
$$$$
G_{2}(\lambda, t) \equiv-\sum_{m>0} \lambda^{2 m}(-1)^{m} e^{-2 t}\left\{J_{2 m+1}(2|\alpha| t)-J_{1-2 m}(2|\alpha| t)\right\}
$$$$
=2 \lambda^{2} \int_{0}^{\pi} \frac{d q}{\pi} e^{-2 t} \sin (2|\alpha| t \sin q)
$$$$
\times\left[\frac{\sin 2 q \cos q}{1+\lambda^{4}+2 \lambda^{2} \cos 2 q}\right] .
$$

To establish these expressions, we have invoked the integral representation of the Bessel functions [16] and the properties of geometric series. With these functions and the help of Eq. (14), the density of particles in the RDS model can now be recast in compact form. Two cases emerge naturally, as follows.

When $\gamma_{e} \gamma_{o}>0$,

$$
\begin{aligned}
\rho(t)-\rho(\infty)= & \frac{\bar{\gamma}}{2 \alpha}\left[F_{1}(\omega, t)+F_{2}(\omega, t)\right] \\
& -\frac{1}{2}\left[F_{1}(1-2 \rho(0), t)+\frac{\bar{\gamma}}{\alpha} F_{2}(1-2 \rho(0), t)\right] .
\end{aligned}
$$

When $\gamma_{e} \gamma_{o}<0$,

$$
\begin{aligned}
\rho(t)-\rho(\infty)= & \frac{\bar{\gamma}}{2|\alpha|}\left[G_{2}(1-2 \rho(0), t)-i G_{1}(\omega, t)\right] \\
& -\frac{1}{2}\left[\frac{\bar{\gamma}}{|\alpha|} G_{2}(\omega, t)+G_{1}(1-2 \rho(0), t)\right] .
\end{aligned}
$$

The case $\gamma_{e} \gamma_{o}=0$ is special and gives rise to a purely exponential time dependence,

$$
\rho(t)=\frac{\bar{\gamma}}{2}+\left(\frac{\bar{\gamma}-2}{4}+\rho(0)\right) e^{-2 t} .
$$

We now proceed with the analysis of the long-time behavior of these expressions. Again, we first consider the case where $\gamma_{e} \gamma_{o}>0$ and then $\gamma_{e} \gamma_{o}<0$.

When $\gamma_{e} \gamma_{o}>0$, the main contribution to the long-time behavior arises from the small $q$ contribution in the expression of the functions $F_{1}$ and $F_{2}$. Therefore, one may expand the integrand of $F_{1}$ and $F_{2}$ in Eq. (B9). It is also essential to pay due attention to the initial condition.

(i) When $0<\gamma_{e} \gamma_{o}<1$ and $0<\rho(0)<1 \quad$ [also $\rho(0) \neq \rho(\infty)$ ], we obtain

$$
\begin{aligned}
\rho(t)-\rho(\infty) \simeq & \frac{1}{4}\left[\frac{\bar{\gamma} \omega}{\alpha\left(1-\omega^{2}\right)}\right. \\
& \left.-\frac{\left(1+\frac{\bar{\gamma}}{\alpha}\right)\{1-2 \rho(0)\}+2 \rho(0)^{2}}{\rho(0)(1-\rho(0))}\right] \frac{e^{-2(1-\alpha) t}}{\alpha t \sqrt{\pi \alpha t}} .
\end{aligned}
$$

(ii) When $0<\gamma_{e} \gamma_{o}<1$ and $\rho(0)=0$, we find

$$
\rho(t)-\rho(\infty) \simeq-\left[1+\frac{\bar{\gamma}}{\alpha}-\left\{\frac{\bar{\gamma} \omega}{\alpha\left(1-\omega^{2}\right)}\right\} \frac{1}{\alpha t}\right] \frac{e^{-2(1-\alpha) t}}{4 \sqrt{\pi \alpha t}} .
$$

(iii) When $0<\gamma_{e} \gamma_{o}<1$ with $\rho(0)=1$, we have

$$
\rho(t)-\rho(\infty) \simeq\left[1-\frac{\bar{\gamma}}{\alpha}+\left\{\frac{\bar{\gamma} \omega}{\alpha\left(1-\omega^{2}\right)}\right\} \frac{1}{\alpha t}\right] \frac{e^{-2(1-\alpha) t}}{4 \sqrt{\pi \alpha t}} .
$$

These results show that for $\gamma_{e} \gamma_{o}>0$, the density generically approaches its stationary value as $\propto t^{-3 / 2} e^{-2(1-\alpha) t}$, with some nontrivial amplitude. Only if the lattice is initially completely empty/occupied by particles is the long-time behavior modified to $\propto t^{-1 / 2} e^{-2(1-\alpha) t}$ (provided $\bar{\gamma} / \alpha \neq \pm 1$ ).

(iv) The case where $\gamma_{e}=\gamma_{o}=\gamma= \pm 1$ is critical and we can check from Eq. (B9) that one recovers the previously known results $[4,18]$,

$$
\rho(t)-\rho(\infty) \simeq \frac{\gamma}{2 \sqrt{\pi t}}
$$

In this case, it is well known $[4,18]$ that the density of particles approaches the steady state algebraically slowly $\left(\propto t^{-1 / 2}\right)$. For $\gamma=1$ (only pair annihilation), the stationary value is $\rho(\infty)=0$; in contrast, we find $\rho(\infty)=1$ for $\gamma=-1$ (only pair creation). We emphasize that for such a critical dynamics, neither the dynamical exponent nor the amplitude of Eq. (B15) depend on the initial condition.

When $\gamma_{e} \gamma_{o}<0$, it is difficult to directly analyze the longtime behavior of the oscillating function $G_{1}$ and $G_{2}$; instead, we seek upper and lower bounds for $\lambda \neq \pm 1$. We observe that the denominator of the integrand in the expressions 
for $G_{1}$ and $G_{2}$ can be bounded as follows: $\left(1-\lambda^{2}\right)^{2} \leqslant(1$ $\left.+\lambda^{4}+2 \lambda^{2} \cos 2 q\right) \leqslant\left(1+\lambda^{2}\right)^{2}$. Therefore, we obtain for the auxiliary functions $G_{1}$ and $G_{2}$

$$
\frac{\lambda}{1+\lambda^{2}} \frac{e^{-2 t} J_{1}(2|\alpha| t)}{2|\alpha| t} \leqslant G_{1}(\lambda, t) \leqslant \frac{\lambda\left(1+\lambda^{2}\right)}{\left(1-\lambda^{2}\right)^{2}} \frac{e^{-2 t} J_{1}(2|\alpha| t)}{2|\alpha| t},
$$

$$
\begin{aligned}
2\left(\frac{\lambda}{1+\lambda^{2}}\right)^{2} \frac{e^{-2 t} J_{2}(2|\alpha| t)}{2|\alpha| t} & \leqslant G_{2}(\lambda, t) \\
& \leqslant 2\left(\frac{\lambda}{1-\lambda^{2}}\right)^{2} \frac{e^{-2 t} J_{2}(2|\alpha| t)}{2|\alpha| t} .
\end{aligned}
$$

If $\lambda= \pm 1$, one has the exact expressions

$$
\begin{aligned}
G_{1}(1, t) & =-G_{1}(-1, t)=e^{-2 t} J_{0}(2|\alpha| t) ; G_{2}(1, t)=G_{2}(-1, t) \\
& =\left(e^{-2 t / 2}|\alpha| t\right) J_{1}(2|\alpha| t) .
\end{aligned}
$$

At long times and for finite $n, \quad e^{-2 t} J_{n}(2|\alpha| t)$ $\simeq\left(e^{-2 t} / \sqrt{\pi|\alpha| t}\right) \cos [2|\alpha| t-(\pi / 4)(2 n+1)]$ and therefore the upper and lower bounds in Eqs. (B16) and (B17) display the same time dependence. With the help of Eq. (B10), we thus deduce the following. (i) When $0<\rho(0)<1$ [and obviously $\rho(0) \neq \rho(\infty)$ ],

$$
\begin{aligned}
\rho(t)-\rho(\infty) \simeq & t^{-3 / 2} e^{-2 t}\left[A \cos \left(2|\alpha| t+\frac{\pi}{4}\right)\right. \\
& \left.+B \cos \left(2|\alpha| t-\frac{\pi}{4}\right)\right],
\end{aligned}
$$

where $A$ and $B$ are some amplitudes depending nontrivially on all the parameters of the system and on the initial density.

(ii) When $\rho(0)=1$, we obtain an explicit expression for the long-time behavior of the density,

$$
\rho(t)-\rho(\infty) \simeq \frac{e^{-2 t}}{2} J_{0}(2|\alpha| t) \simeq \frac{e^{-2 t}}{2 \sqrt{\pi|\alpha| t}} \cos \left(2|\alpha| t-\frac{\pi}{4}\right) .
$$

(iii) When $\rho(0)=0$, we also have an explicit expression for the long-time behavior of the density,

$$
\rho(t)-\rho(\infty) \simeq-\frac{e^{-2 t}}{2} J_{0}(2|\alpha| t) \simeq \frac{e^{-2 t}}{2 \sqrt{\pi|\alpha| t}} \cos \left(2|\alpha| t+\frac{3 \pi}{4}\right) .
$$

These results show that, for $\gamma_{e} \gamma_{o}<0$, the density displays oscillations which are damped by a factor $t^{-\beta} e^{-2 t}$, where $\beta$ $=3 / 2$ for generic initial densities $\rho(0)$, with two exceptions: we have $\beta=1 / 2$ if the system is initially completely empty or occupied.

Of course, following the same approach, one would be able to compute every $n$-point correlation function for both the KISC and RDS. While perfectly straightforward, these computations become rather tedious.
[1] B. Schmittmann and R. K. P. Zia, in Phase Transitions and Critical Phenomena, edited by C. Domb and J. L. Lebowitz (Academic Press, New York, 1995) Vol. 17; D. Mukamel, in Soft and Fragile Matter: Nonequilibrium Dynamics, Metastability and Flow, edited by M. E. Cates and M. R. Evans (IOP Publishing, Bristol, 2000).

[2] Nonequilibrium Statistical Mechanics in One Dimension, edited by V. Privman (Cambridge University Press, Cambridge, 1997).

[3] G. M. Schütz, in Phase Transitions and Critical Phenomena, edited by C. Domb and J. Lebowitz (Academic Press, London, 2000), Vol. 19.

[4] A. A. Lushnikov, Sov. Phys. JETP 64, 811 (1986); M. D. Grynberg, T. J. Newman, and R. B. Stinchcombe, Phys. Rev. E 50, 957 (1994); M. D. Grynberg and R. B. Stinchcombe, Phys. Rev. Lett. 74, 1242 (1995); Phys. Rev. E 52, 6013 (1995); Phys. Rev. Lett. 76, 851 (1996); P.-A. Bares and M. Mobilia, Phys. Rev. E 59, 1996 (1999); Phys. Rev. Lett. 83, 5214 (1999); S.-C. Park, J.-M. Park, and D. Kim, ibid. 85, 892 (2000); P.-A. Bares and M. Mobilia, ibid. 85, 893 (2000).

[5] R. J. Glauber, J. Math. Phys. 44, 294 (1963).

[6] Z. Ràcz, Phys. Rev. Lett. 55, 1707 (1985).

[7] F. Family and J. G. Amar, J. Stat. Phys. 55, 1235 (1991).
[8] Z. Ràcz and R. K. P. Zia, Phys. Rev. E 49, 139 (1994).

[9] B. Schmittmann and F. Schmüser, Phys. Rev. E 66, 046130 (2002).

[10] F. Schmüser and B. Schmittmann, J. Phys. A 35, 2569 (2002).

[11] M. Mobilia, R. K. P. Zia, and B. Schmittmann, J. Phys. A 37, L407 (2004).

[12] N. Kuroda, M. Nishida, Y. Tabata, Y. Wakabayashi, and K. Sasaki, Phys. Rev. B 61, 11217 (2000).

[13] N. Kuroda, Y. Wakabayashi, M. Nishida, N. Wakabayashi, M. Yamashita, and N. Matsushita, Phys. Rev. Lett. 79, 2510 (1997); N. Kuroda, Y. Tabata, M. Nishida, and M. Yamashita Phys. Rev. B 59, 12973 (1999); Y. Tabata and N. Kuroda, Synth. Met. 101, 329 (1999); Y. Tabata and N. Kuroda, Phys. Rev. B 61, 3085 (2000); H. Tanaka, K. Marumoto, S. Kuroda, and M. Yamashita, Synth. Met. 101, 135 (2003).

[14] R. Kroon, H. Fleurent, and R. Sprik, Phys. Rev. E 47, 2462 (1993); R. Kopelman, C. S. Li, and R. Sprik, J. Lumin. 45, 40 (1990).

[15] M. A. Aliev, Physica A 277, 261 (2000).

[16] M. Abramowitz and I. Stegun, Handbook of Mathematical Functions (Dover, New York, 1965).

[17] C. Itzykson and J.-M. Drouffe, Statistical Field Theory (Cambridge University Press, Cambridge, 1989), Vol. 1.

[18] M. Mobilia, Phys. Rev. E 65, 046127 (2002); M. Mobilia and 
P.-A. Bares, ibid. 63, 056112 (2001); M. Mobilia, Ph.D. thesis, EPFL N. 2552 (2002).

[19] J. R. G. Mendoça and M. J. de Oliveira, J. Stat. Phys. 78, 1429 (1998).

[20] J. E. Santos, J. Phys. A 30, 3249 (1997).
[21] B. U. Felderhof, Rep. Math. Phys. 1, 215 (1971); 2, 151 (1971).

[22] N. N. Bogoliubov, Studies in Statistical Mechanics, edited by J. de Boer and E. Uhlenbeck (North-Holland, Amsterdam, 1962), Vol. 1. 